\title{
Dynamics of starbursting dwarf galaxies. II. UGC 4483
}

\author{
F. Lelli ${ }^{1}$, M. Verheijen ${ }^{1}$, F. Fraternali ${ }^{1,2}$, and R. Sancisi ${ }^{1,3}$ \\ 1 Kapteyn Astronomical Institute, University of Groningen, Postbus 800, 9700 AV Groningen, The Netherlands \\ e-mail: lelli@astro.rug.nl \\ 2 Department of Astronomy, University of Bologna, via Ranzani 1, 40127 Bologna, Italy \\ 3 INAF - Astronomical Observatory of Bologna, via Ranzani 1, 40127 Bologna, Italy
}

Received 23 April 2012 / Accepted 10 July 2012

\begin{abstract}
UGC 4483 is a nearby blue compact dwarf (BCD) galaxy. HST observations have resolved the galaxy into single stars and this has led to the derivation of its star formation history and to a direct estimate of its stellar mass. We have analysed archival VLA observations of the 21-cm line and found that UGC 4483 has a steeply-rising rotation curve which flattens in the outer parts at a velocity of $\sim 20 \mathrm{~km} \mathrm{~s}{ }^{-1}$. Radial motions of $\sim 5 \mathrm{~km} \mathrm{~s}^{-1}$ may also be present. As far as we know, UGC 4483 is the lowest-mass galaxy with a differentially rotating H I disk. The steep rise of the rotation curve indicates that there is a strong central concentration of mass. We have built mass models using the HST information on the stellar mass to break the disk-halo degeneracy: old stars contribute $\sim 50 \%$ of the observed rotation velocity at 2.2 disk scale-lengths. Baryons (gas and stars) constitute an important fraction of the total dynamical mass. These are striking differences with respect to typical dwarf irregular galaxies (dIrrs), which usually have slowly-rising rotation curves and are thought to be entirely dominated by dark matter. BCDs appear to be different from non-starbursting dIrrs in terms of their HI and stellar distributions and their internal dynamics. To their high central surface brightnesses and high central H I densities correspond strong central rotation-velocity gradients. This implies that the starburst is closely related with the gravitational potential and the concentration of gas. We discuss the implications of our results on the properties of the progenitors/descendants of BCDs.
\end{abstract}

Key words. dark matter - galaxies: individual: UGC 4483 - galaxies: dwarf - galaxies: starburst - galaxies: evolution galaxies: kinematics and dynamics

\section{Introduction}

The mechanisms that trigger strong bursts of star formation in galaxies are poorly understood. In the Local Universe, starburst activity is mostly observed in low-mass galaxies, which are usually classified as blue compact dwarfs (BCDs; e.g. Gil de Paz et al. 2003), amorphous dwarfs (e.g. Gallagher \& Hunter 1987), or H II galaxies (e.g. Taylor et al. 1995). Hereafter, we will refer to any starbursting dwarf galaxy as a BCD. Several studies (e.g. Gil de Paz \& Madore 2005; Tosi 2009, and references therein) have shown that BCDs are not young galaxies undergoing their first burst of star formation (as suggested by Searle \& Sargent 1972), as they also contain old stellar populations with ages >2-3 Gyr. In particular, Hubble Space Telescope (HST) has made it possible to resolve nearby BCDs into single stars and to derive color-magnitude diagrams deep enough to provide the following information: i) accurate distances of the galaxies, ii) a direct estimate of their total stellar mass, and iii) their star formation history (SFH; e.g. Tosi 2009). These SFHs show that the starburst is a short-lived phenomenon, typically sustained for a few $10^{8} \mathrm{yr}$ (McQuinn et al. 2010a). Thus, BCDs are transition-type dwarfs but the nature of their progenitors and descendants remains unclear. In particular, it is not known whether there are evolutionary connections with dwarf irregulars (dIrrs), spheroidals (dSphs), and/or ellipticals (dEs; e.g. Papaderos et al. 1996; van Zee et al. 2001).

There are striking differences between BCDs and other types of dwarf galaxies: i) the old stellar component of BCDs generally has a smaller scale-length and higher central surface brightness than dIrrs and dEs/dSphs (e.g. Papaderos et al. 1996; Gil de Paz \& Madore 2005); ii) BCDs have strong concentrations of H I within the starburst region, where the column densities are typically 2-3 times higher than in dIrrs (e.g. van Zee et al. 1998, 2001); iii) BCDs have steep central velocity gradients that are not observed in dIrrs (e.g. van Zee et al. 1998, 2001). The steep velocity gradients may signify a steeply-rising rotation curve (van Zee et al. 2001; Lelli et al. 2012), high velocity dispersion, or non-circular motions (e.g. Elson et al. 2011b). Detailed studies of the gas kinematics are needed to determine the inner shape of the rotation curve. Recently, Lelli et al. (2012) studied the BCD prototype I Zw 18 and found that it has a flat rotation curve with a steep rise in the inner parts, indicating that there is a high central concentration of mass. Such a mass concentration is not observed in typical dIrrs. This points to a close connection between the starburst and the gravitational potential. It is also clear that a BCD like I Zw 18 cannot evolve into a typical dIrrs at the end of the starburst, unless the central concentration of mass is removed. It is important, therefore, to investigate whether all BCDs have steeply-rising rotation curves, and to determine the relative contributions of gas, stars and dark matter to the gravitational potential.

We present a detailed study of the gas kinematics of UGC 4483, a starbursting dwarf galaxy located in the M 81 group and resolved into individual stars by HST (Dolphin et al. 2001; Izotov \& Thuan 2002). UGC 4483 is extremely metal-poor $(12+\log (\mathrm{O} / \mathrm{H}) \simeq 7.5$, see Skillman et al. 1994; van Zee \& Haynes 2006) and may be classified as a "cometary" $\mathrm{BCD}$, as its high-surface-brightness starburst region is located 


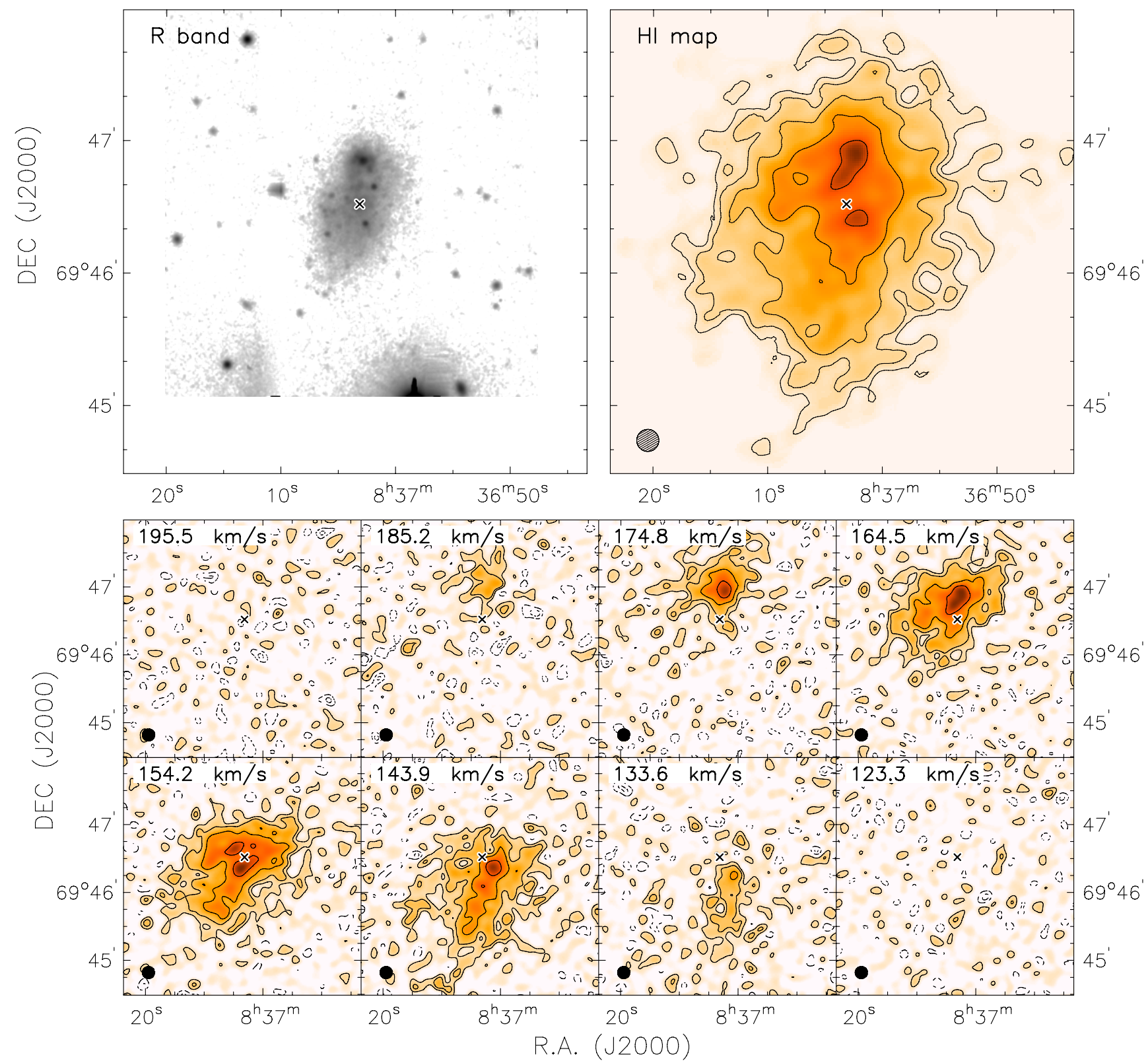

Fig. 1. Top-left: $R$-band image (from Gil de Paz et al. 2003). Top-right: total HI map at 10" resolution. The contour values are 1.7, 3.4, 6.8, 13.6, $27.2 \times 10^{20}$ atoms cm $\mathrm{cm}^{-2}$. Bottom: channel maps at 10" resolution. Contours are at 1.5, 3, 6, 12, $24 \times \sigma$, where $\sigma=0.66 \mathrm{mJy} / \mathrm{beam}(\sim 2 \times$ $10^{19}$ atoms $\mathrm{cm}^{-2}$ ). In each panel, the cross and the circle show the galaxy center and the beam size, respectively.

at the edge of an elongated low-surface-brightness stellar body (see Fig. 1, top-left). Previous H I studies (Lo et al. 1993; van Zee et al. 1998) showed that the galaxy has an extended H I disk, with a strong H I concentration near the starburst region and a steep central velocity gradient, but the H I kinematics was not studied in detail. We analysed archival VLA data and were able to derive a rotation curve which we used to investigate the distributions of luminous and dark matter in this galaxy.

\section{Data reduction and analysis}

We analysed HI data taken from the VLA archive. The observations have been carried out with the B and C arrays, and are described in van Zee et al. (1998). The correlator was used in $2 \mathrm{AD}$ mode, with a total bandwidth of $1.56 \mathrm{MHz}$ $\left(\sim 330 \mathrm{~km} \mathrm{~s}^{-1}\right)$. An on-line Hanning taper was applied to the data, producing 127 spectral line channels with a width of $12.3 \mathrm{kHz}$ $\left(\sim 2.6 \mathrm{~km} \mathrm{~s}^{-1}\right)$.

We interactively flagged, calibrated, and combined the raw UV data using the AIPS package and following standard VLA procedures. The UV data were mapped using a robust weighting technique (Briggs 1995) and a Gaussian baseline taper to attenuate the longest baselines. After various trials, we chose a robust parameter of -1 and a taper full width at half maximum (FWHM) of $40 \mathrm{k} \lambda$; these parameters minimize sidelobes and wings in the beam profile and lead to a datacube with an angular resolution of $5 . .7 \times 4$.' 5 .

After the Fourier transform, the data analysis was continued using the Groningen Imaging Processing SYstem (GIPSY; van der Hulst et al. 1992). A continuum map was constructed by averaging line-free channels, and subtracted from the datacube. The channel maps were cleaned (Högbom 1974) down to $0.3 \sigma$, using a mask to define the search areas for the 
F. Lelli et al.: Dynamics of starbursting dwarf galaxies. II.

clean-components that were then restored with a Gaussian beam of the same FWHM as the antenna pattern. The mask was constructed by smoothing the datacube both in velocity (to $10.4 \mathrm{~km} \mathrm{~s}^{-1}$ ) and spatially (to $20^{\prime \prime}$ ) and clipping at $\sim 3 \sigma_{\mathrm{s}}$ (where $\sigma_{\mathrm{s}}$ is the rms noise in the smoothed cube). To improve the signal-to-noise ratio, the cleaned datacube was smoothed in velocity to a resolution of $5.2 \mathrm{~km} \mathrm{~s}^{-1}$ and spatially to $10^{\prime \prime}$, providing a $3 \sigma$ column density sensitivity of $6 \times 10^{19}$ atoms cm ${ }^{-2}$ per $2.6 \mathrm{~km} \mathrm{~s}^{-1}$-wide channel.

A total H I map was constructed by summing the signal inside the clean-mask; a pseudo- $3 \sigma$ contour was calculated following Verheijen \& Sancisi (2001). A velocity field was derived by fitting a Gaussian function to the HI line profiles. Fitted Gaussians with a peak intensity less than $3 \sigma$ and a FWHM smaller than $5.2 \mathrm{~km} \mathrm{~s}^{-1}$ were discarded. The H I line profiles are quite broad and asymmetric, thus the velocity field derived from the Gaussian fitting provides only an overall description of the galaxy kinematics. Our kinematical analysis is based on threedimensional (3D) models of the observations (Sect. 3.2) and not merely on the two-dimensional (2D) velocity field.

\section{Results}

\subsection{HI distribution and kinematics}

Figure 1 shows the total H I map of UGC 4483 at a resolution of $10^{\prime \prime}$ (top-right) and a $R$-band image at the same scale (top-left). The H I distribution is lopsided and closely resembles the optical morphology. There is a strong $\mathrm{H}$ I concentration near the starburst region to the North. At $5^{\prime \prime} .7 \times 4^{\prime \prime} .5$ resolution $(\sim 80 \mathrm{pc})$, the peak column densities are $\sim 5 \times 10^{21}$ atoms cm $\mathrm{cm}^{-2}$ ( $\sim 40 M_{\odot} \mathrm{pc}^{-2}$ ) (same as found by van Zee et al. 1998).

The H I kinematics of UGC 4483 is illustrated in Fig. 1 (bottom) and Fig. 2 (top). The $\mathrm{H} \mathrm{I}$ emission shows a velocity gradient along a position angle $\mathrm{PA} \sim 0^{\circ}$, which roughly corresponds to the optical major axis of the galaxy, suggesting that there is a rotating $\mathrm{HI}$ disk. The velocity field indicates large-scale differential rotation. It also shows, however, large-scale asymmetries. In the next section, we derive the rotation curve of UGC 4483 and discuss possible non-circular motions.

\subsection{Rotation curve and non-circular motions}

Rotation curves of disk galaxies are usually derived by fitting a tilted-ring model to a velocity field (e.g. Begeman 1987). In the case of UGC 4483, there are severe limitations because of the asymmetries in the velocity field. Van Zee et al. (1998) modeled the velocity field of UGC 4483 and obtained a rough estimate of the dynamical mass. We derived a rotation curve using the following approach. As a first step, we fit a tilted-ring model to the velocity field to obtain an initial estimate of the rotation curve. Then, this rotation curve was used as input to build a 3D kinematic model and subsequently corrected by trial and error to produce a model-cube that matches the observations.

When fitting a tilted-ring model to the velocity field, we used a ring width of $10^{\prime \prime}$ ( 1 beam), thus the points of the rotation curve are nearly independent. The points of the velocity field were weighted by $\cos ^{2}(\theta)$, where $\theta$ is the azimuthal angle in the plane of the galaxy. We kept the center $\left(x_{0}, y_{0}\right)$ and the inclination $i$ fixed, using the values derived from the optical image by fitting ellipses to the outermost isophotes (see Table 1). An inclination of $58^{\circ}$ is formally a lower limit, because the stellar component may be thick; if the disk is assumed to be $5^{\circ}$ more edge-on, the rotation velocities would decrease by only $\sim 5 \%$. We also fixed
Table 1. Properties of UGC 4483.

\begin{tabular}{lc}
\hline \hline$\alpha(\mathrm{J} 2000)$ & $08^{\mathrm{h}} 37^{\mathrm{m}} 3 .^{\mathrm{s}} 1 \pm 0 .{ }^{\mathrm{s}} 5$ \\
$\delta(\mathrm{J} 2000)$ & $69^{\circ} 46^{\prime} 31^{\prime \prime} \pm 2^{\prime \prime}$ \\
Distance $(\mathrm{Mpc})$ & $3.2 \pm 0.2$ \\
$V_{\text {sys }}\left(\mathrm{km} \mathrm{s}^{-1}\right)$ & $158 \pm 2$ \\
Position angle $\left(^{\circ}\right)$ & $0 \pm 5$ \\
Inclination angle $\left(^{\circ}\right)$ & $58 \pm 3$ \\
$V_{\text {rot }}\left(\mathrm{km} \mathrm{s}^{-1}\right)$ & $19 \pm 2$ \\
$M_{\text {dyn }}\left(10^{7} M_{\odot}\right)$ & $16 \pm 3$ \\
$M_{*}\left(10^{7} M_{\odot}\right)$ & $1.0 \pm 0.3$ \\
$M_{\mathrm{HI}}\left(10^{7} M_{\odot}\right)$ & $2.5 \pm 0.3$ \\
$L_{\mathrm{B}}\left(10^{7} L_{\odot}\right)$ & 1.4 \\
$L_{R}\left(10^{7} L_{\odot}\right)$ & 0.9 \\
\hline
\end{tabular}

Notes. Luminosities were calculated using the apparent magnitudes from Gil de Paz et al. (2003), the distance from Dolphin et al. (2001) and the solar absolute magnitudes from Binney \& Merrifield (1998). The stellar mass was calculated integrating the SFH from McQuinn et al. (2010a) and assuming a gas recycling efficiency of $30 \%$. The dynamical mass was calculated taking into account the pressure-support.

the PA assuming the value of $0^{\circ}$ suggested by the H I morphology (Fig. 1, top), which is consistent with the optical PA $\left(\simeq-10^{\circ}\right)$ within the uncertainties. As a first step, we determined the systemic velocity $V_{\text {sys }}$, by taking the mean value over all the rings. Then, we fixed $V_{\text {sys }}$ and determined the rotation velocity $V_{\text {rot }}$ and the radial velocity $V_{\text {rad }}$ for each ring. The rotation curve has an amplitude of $\sim 18-20 \mathrm{~km} \mathrm{~s}^{-1}$ (Fig. 4), while the radial velocities are $\sim 4-5 \mathrm{~km} \mathrm{~s}^{-1}$.

As a final step in the derivation of the rotation curve, we built 3D kinematic models, similarly to Swaters et al. (2009) and Lelli et al. $(2010,2012)$. The disk kinematics is assumed to be axisymmetric, while the H I distribution is clumpy, i.e. the surface density varies with position as in the observed H I map. The procedure is as follows: i) a disk with uniform surface density and constant thickness is constructed by fixing the velocity dispersion $\sigma_{\mathrm{HI}}$, the rotation velocity $V_{\text {rot }}$ and the radial velocity $V_{\text {rad }}$ at every radius; ii) the disk is projected on the sky using the geometrical parameters $\left(x_{0}, y_{0}\right), V_{\text {sys }}, \mathrm{PA}$, and $i$ and a model-cube is created; iii) the model-cube is convolved with the observational beam; and iv) the HI line profiles are rescaled to reproduce the observed HI map, i.e. the flux density is recovered at every spatial pixel. For the geometrical parameters, we used the values in Table 1 . For the vertical distribution, we assumed an exponential-law $\exp \left(-z / z_{0}\right)$ with $z_{0}=100 \mathrm{pc}$. We also assumed that $\sigma_{\mathrm{HI}}=8 \mathrm{~km} \mathrm{~s}^{-1}$ over the entire disk; a mean velocity dispersion higher than $10 \mathrm{~km} \mathrm{~s}^{-1}$ can be ruled out by comparing the shape of position-velocity (PV) diagrams obtained from the models and the observations. The values of $z_{0}$ and $\sigma_{\mathrm{HI}}$ are slightly degenerate, but do not significantly affect the final results. For the rotation curve, we used the rotation velocities obtained by fitting the velocity field, then we corrected them by trial and error to obtain a modelcube that matches the observations. We find a good match by increasing the first four points by $\sim 3 \mathrm{~km} \mathrm{~s}^{-1}$. This rotation curve is shown in Fig. 4. The comparison between models and observations has been done using velocity fields (see Fig. 2) and PV-diagrams (see Fig. 3). Note that the model velocity fields were derived from the model-cubes and, therefore, include the effects of spatial and spectral resolution, velocity 


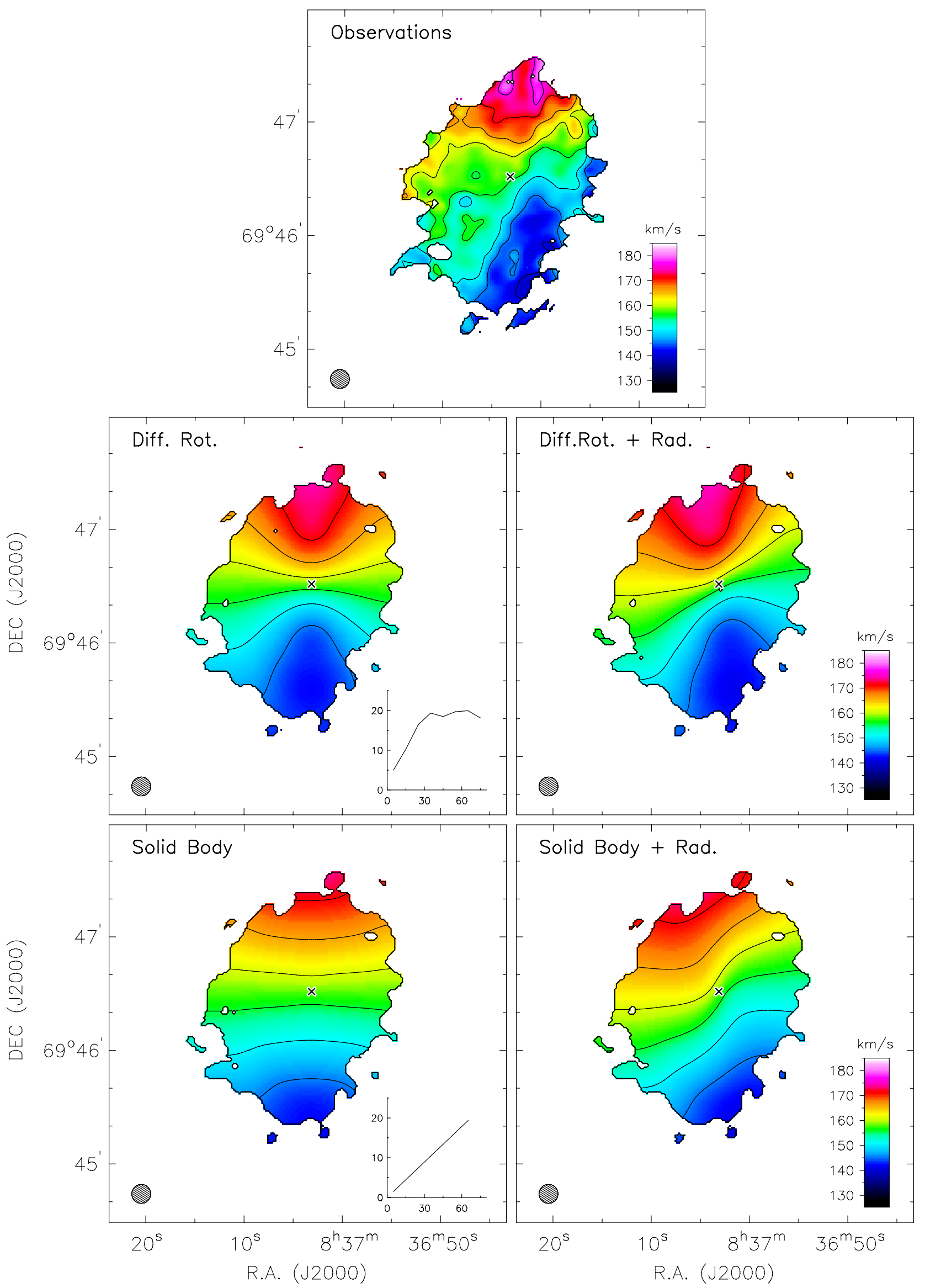

Fig. 2. Velocity fields derived from different 3D kinematic models and the observations. The rotation curve used to build the models is shown in the inset on the left ( $x$-axis: radius in arcsec, $y$-axis: rotation velocity in $\mathrm{km} \mathrm{s}^{-1}$ ). Contours range from 140 to $181.6 \mathrm{~km} \mathrm{~s}^{-1}$, with steps of $5.2 \mathrm{~km} \mathrm{~s}^{-1}$. The circles show the beam size (10"). See Sect. 3.1 for details. 


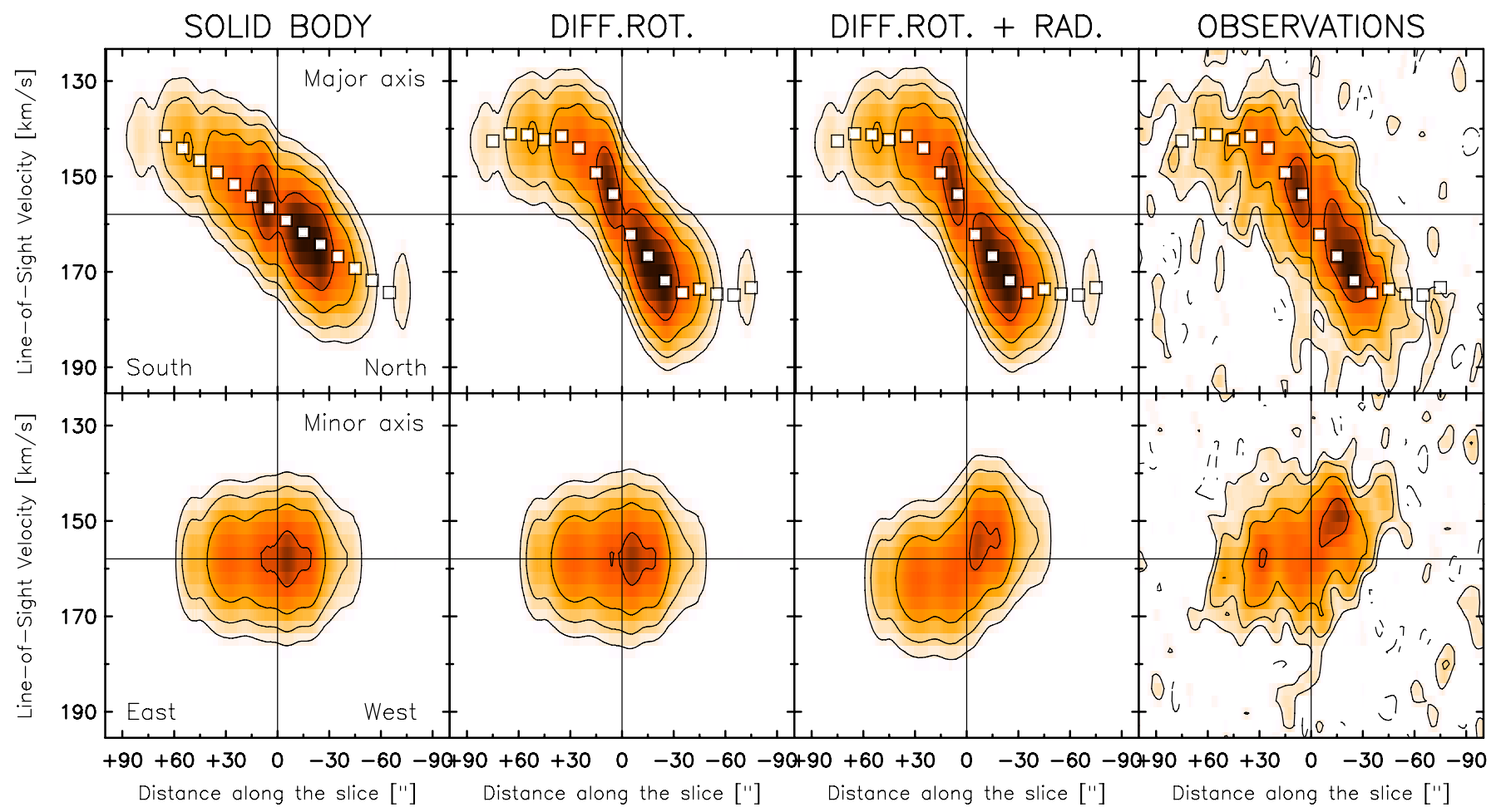

Fig. 3. Position-velocity diagrams derived for different 3D kinematic models and from the observations. The slices are taken along the major and minor axes. Contours are at 1.5, 3, 6, $12 \times \sigma$, where $\sigma=0.66 \mathrm{mJy} /$ beam. Squares show the rotation curve used to build the models. See Sect. 3.1 for details.

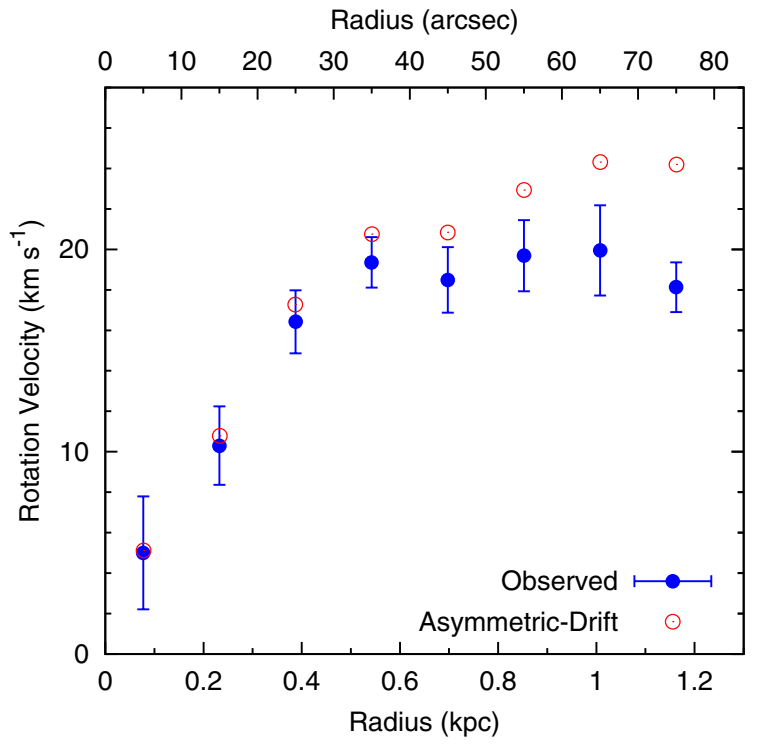

Fig. 4. Observed H I rotation curve (blue-filled circles) and asymmetricdrift-corrected rotation curve (red-open circles).

dispersion, and possible non-circular motions. Because in our models we initially assumed a uniform HI disk, some aspects of the effects of beam-smearing are not fully included if the $\mathrm{H}$ I distribution varies rapidly on small scales. However, we used PV-diagrams at the full resolution $\left(5^{\prime \prime} .7 \times 44^{\prime \prime} 5\right)$ and compared them with those at $10^{\prime \prime}$ to check that beam-smearing effects do not significantly affect our rotation curve.

Figure 4 (filled-circles) shows that UGC 4483 has a steeplyrising rotation curve that flattens in the outer parts at a velocity of $\sim 18-20 \mathrm{~km} \mathrm{~s}^{-1}$. This is a striking difference with respect to
dIrrs of similar mass, as they usually have a slowly-rising rotation curve close to a solid body. As far as we know, UGC 4483 is the lowest-mass galaxy with a differentially rotating $\mathrm{H}$ I disk. To further test the validity of this result, we built 3D kinematic models assuming a solid-body rotation curve and compared them with the differentially-rotating disk-model and with the observations. The results are shown in Figs. 2 and 3. It is clear that a flat rotation curve provides a better match of the observations than a solid-body one, as the iso-velocity contours of the observed velocity field display a curvature that is typical of differential rotation. The same conclusions are reached by comparing PV-diagrams obtained from the models and the observations along the major axis (Fig. 3, top). The observed PV-diagram shows a "flattening" on the southern-approaching side of the disk, that is reproduced only by a differentially rotating disk. On the northern-receding side, the "flattening" is less clear as the disk is less extended, but a differentially rotating disk is still preferable than a solid body one.

A simple rotating disk, however, cannot reproduce the asymmetries present in the observed velocity field. In particular, the kinematic minor axis, defined by the contours close to the systemic velocity, is not orthogonal to the major axis. This may be due either to radial motions (e.g. Fraternali et al. 2002) or to an oval distortion of the gravitational potential (e.g. Bosma 1978). These asymmetries can be seen also in the PV-diagram taken along the geometrical minor axis, i.e. in the direction perpendicular to the major axis (Fig. 3, bottom). Thus, we improved our models by adding a constant radial component of $\sim 5 \mathrm{~km} \mathrm{~s}^{-1}$, which is also indicated by the tilted-ring fit to the velocity field. A model with solid-body rotation plus radial motions is still not acceptable (Fig. 2, bottom-right), whereas a model with differential rotation plus radial motions reproduces most of the features in the data (Fig. 2, middle-right and Fig. 3, bottom). 


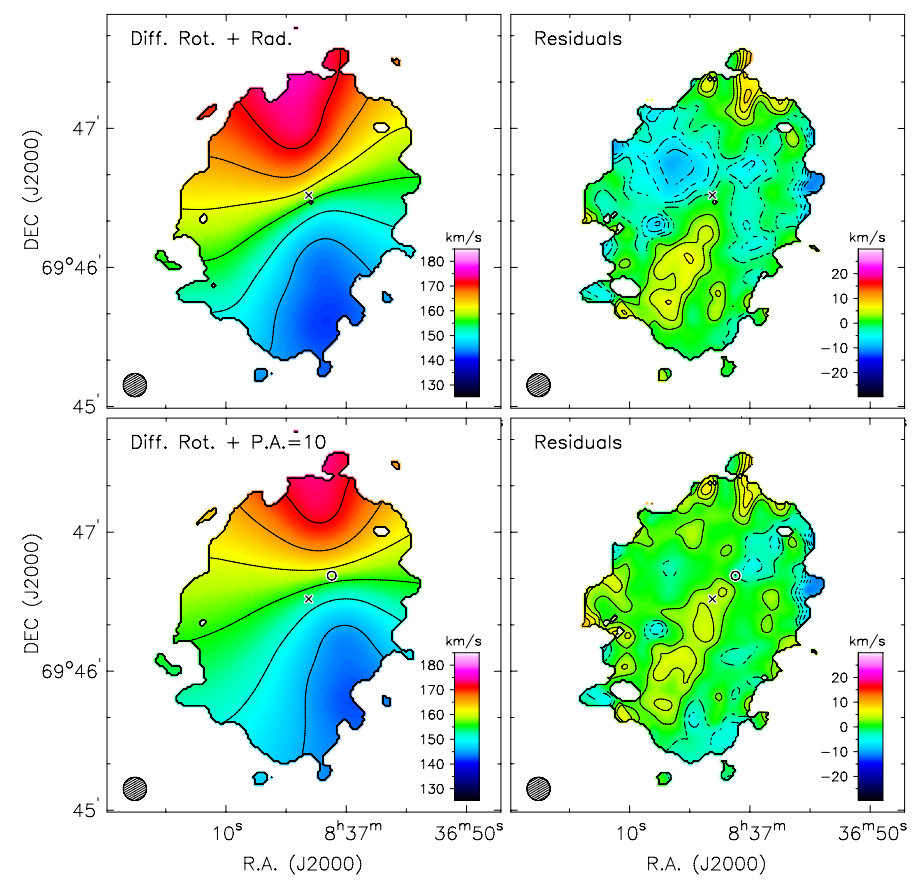

Fig. 5. Left: velocity fields derived from disk-models with different geometrical parameters: optical center (cross), $\mathrm{PA}=0^{\circ}$, and radial motions of $\sim 5 \mathrm{~km} \mathrm{~s}^{-1}$ (top panel, same as Fig. 2 middle-right); shifted dynamical center (circle), $\mathrm{PA}=10^{\circ}$, and no radial motions (bottom panel). Contours are the same as in Fig. 2. Right: differences between the observed and the model velocity fields. Contours are at $\pm 2, \pm 4, \pm 6$, $\pm 8 \mathrm{~km} \mathrm{~s}^{-1}$. The filled circle shows the beam.

The errors on the rotation curve have been estimated as $\sigma_{\text {rot }}^{2}=\sigma_{\text {fit }}^{2}+\sigma_{\text {asym }}^{2}$, where $\sigma_{\text {fit }}^{2}$ is the formal error given by the tilted-ring fit and $\sigma_{\text {asym }}^{2}$ is an additional uncertainty due to the asymmetries between the approaching and receding sides, that is estimated as $\sigma_{\text {asym }}=\left(V_{\text {rot,app }}-V_{\text {rot,rec }}\right) / 4$ (Swaters et al. 2009). We point out that the observed H I line profiles are quite broad and, therefore, it is very difficult to trace the rotation curve in the innermost parts. We compared models that have an inner solid-body rise and flatten at different radii and estimated that the rotation curve must flatten between $\sim 0.3$ and $\sim 0.6 \mathrm{kpc}$, giving an inner rotation-velocity gradient between $\sim 35$ and $\sim 65 \mathrm{~km} \mathrm{~s}^{-1} \mathrm{kpc}^{-1}$. We adopted an intermediate value for the rotation-velocity gradient.

In UGC 4483 the $\mathrm{HI}$ velocity dispersion $\sigma_{\mathrm{HI}}$ is only a factor $\sim 2-3$ smaller than the observed rotation velocity $V_{\text {rot }}$. Thus, to trace the gravitational potential, the rotation curve has to be corrected for pressure support. We calculated the asymmetricdrift correction following Meurer et al. (1996). We assumed that the HI disk has constant scale-height and velocity dispersion, and fitted the H I surface density profile (Fig. 6) with the Gaussian function $\Sigma_{\mathrm{HI}}(R)=\Sigma_{0} \times \exp \left(-R^{2} / 2 s^{2}\right)$, obtaining $\Sigma_{0}=10.5 M_{\odot} \mathrm{pc}^{-2}$ and $s=580 \mathrm{pc}$. The circular velocity $V_{\text {circ }}$, corrected for asymmetric-drift, is thus given by $V_{\text {circ }}^{2}=V_{\text {rot }}^{2}+\sigma_{\mathrm{HI}}^{2}\left(R^{2} / s^{2}\right)$. Using the 3D models, we can constrain the mean velocity dispersion between $\sim 6$ and $\sim 10 \mathrm{~km} \mathrm{~s}^{-1}$. We assumed the intermediate value of $8 \mathrm{~km} \mathrm{~s}^{-1}$. The asymmetricdrift correction is significant only in the outer parts (see Fig. 4). Depending on the assumed value of $\sigma_{\mathrm{HI}}$, the correction at the last measured points may be between $\sim 2$ and $\sim 8 \mathrm{~km} \mathrm{~s}^{-1}$, giving a dynamical mass between about 1 and $2 \times 10^{8} M_{\odot}$.

In the above analysis, we used the center and the PA derived from the stellar and H I morphologies. However, the differences

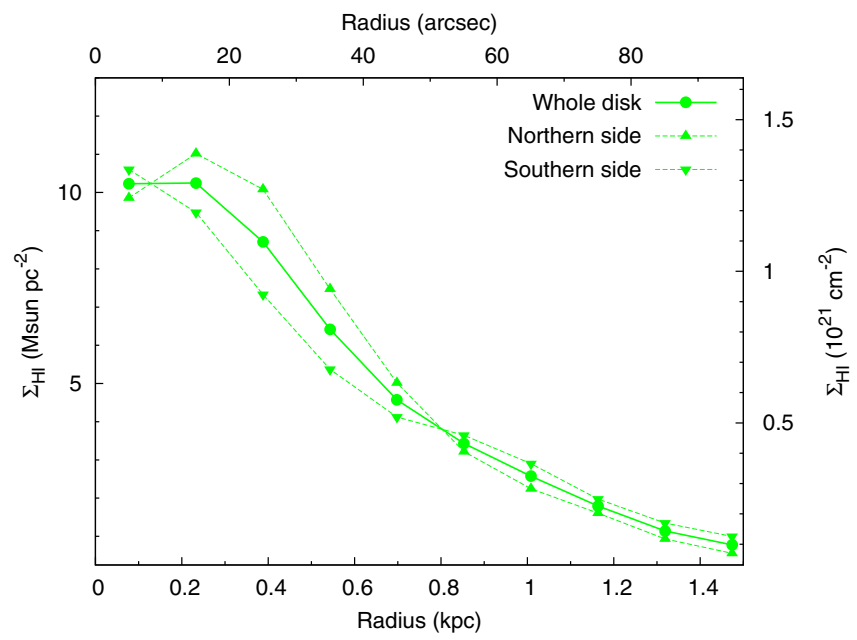

Fig. 6. H I surface density profiles (inclination corrected), derived separately for the northern (up-triangles) and southern (down-triangles) sides and for the entire galaxy (dots).

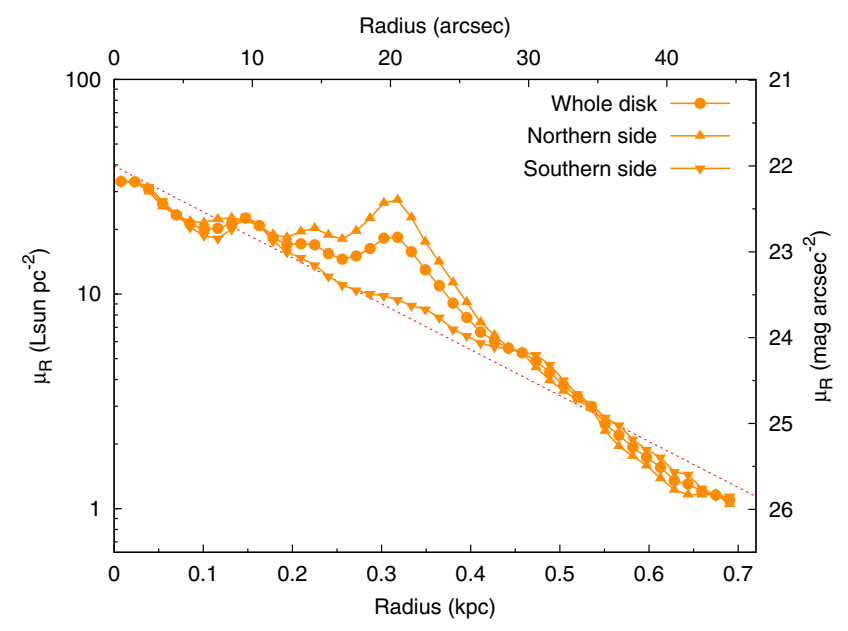

Fig. 7. $R$-band surface brightness profiles, derived separately for the northern (up-triangles) and southern (down-triangles) sides and for the entire galaxy (dots). The profiles are not corrected for internal extinction and inclination. The red-dotted line shows an exponential fit to the southern profile, giving $\mu_{0}=22.0 \mathrm{mag} \operatorname{arcsec}^{-2}\left(\sim 40 L_{\odot} \mathrm{pc}^{-2}\right)$ and $R_{0}=13 \cdot 1^{\prime \prime} \pm 0.1^{\prime \prime}(\sim 203 \mathrm{pc})$.

between the observed and the best-model velocity fields (Fig. 5, top-right) show a systematic pattern that may point to a different location of the dynamical center (cf. Warner et al. 1973, their Fig. 8). Thus, we built further models by changing the position of the center and found that a shift of $\sim 14^{\prime \prime}(\sim 220 \mathrm{pc})$ towards the North-West reduces the pattern significantly. Moreover, a disk-model with an off-set center and a $\mathrm{PA} \simeq 10^{\circ}$ can reproduce the observed velocity field without radial motions (Fig. 5, bottom). However, these values for the center and the PA are in marked contrast with the observed stellar and H I morphologies. In particular, the distribution of the old stars (which constitute an important fraction of the dynamical mass in the inner parts, see Sect. 3.3) is remarkably symmetric and consistent with the center and PA previously adopted (see Fig. 6 of Dolphin et al. 2001; and Fig. 9 of Izotov \& Thuan 2002). Thus, in the following, we keep the dynamical center coincident with the optical one. This has no significant effects on the derived rotation curve. 


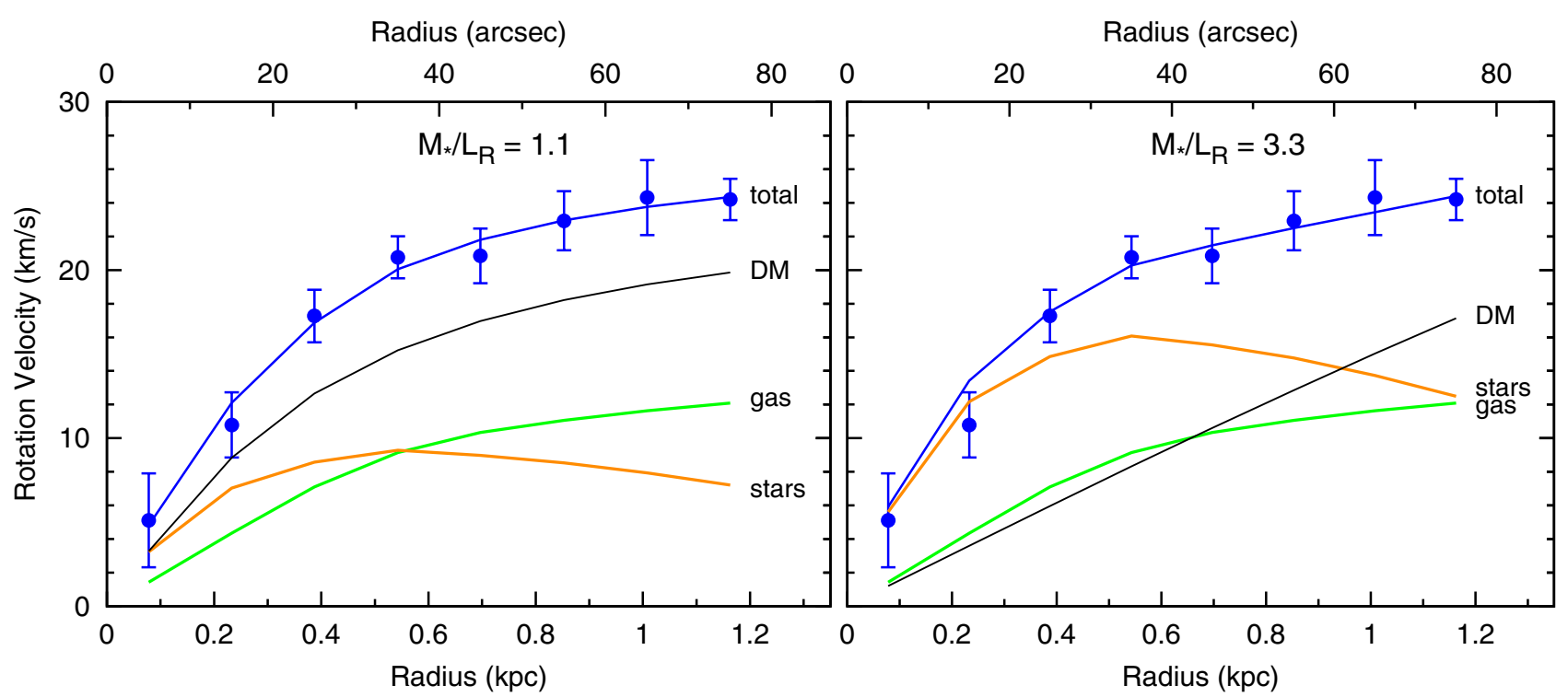

Fig. 8. Rotation curve decompositions with the $M_{*} / L_{R}(=1.1)$ obtained from the study of the resolved stellar populations $(l e f t)$ and with the $M_{*} / L_{R}$ $(=3.3)$ from the maximum-disk solution (right). Dots show the observed rotation curve. Lines show the gravitational contribution of the gas, stars, and dark matter and the resulting rotation curve.

\subsection{Mass models}

In Sect. 3.2, we showed that UGC 4483 has a steeply-rising and flat rotation curve, indicating that there is a strong central concentration of mass. To determine the relative contributions of luminous and dark matter to the gravitational potential, we built mass models following Begeman (1987).

The gravitational contribution of the stars was calculated using a new approach: we estimated the stellar mass-to-light ratio $M_{*} / L$ using the HST results on the resolved stellar populations. Surface brightness profiles were derived from a skysubtracted $R$-band image (Fig. 1, top-left), azimuthally averaging over a set of ellipses defined by $\left(x_{0}, y_{0}\right), i$, and PA (see Table 1). The sky-background was determined by masking the sources in the field and fitting a 2D polynomial to the masked image. The surface brightness profiles were not corrected for internal extinction, as UGC 4483 is extremely metal-poor (Skillman et al. 1994) and the dust content is likely to be low, but were corrected for Galactic extinction assuming $A_{R}=0.09$ (Schlegel et al. 1998). Figure 7 shows the surface brightness profiles derived for the entire galaxy and for the southern (approaching) and northern (receding) halves separately. At $R \simeq 20^{\prime \prime}$, the northern side of the galaxy is $\sim 1$ mag brighter than the southern one, owing to the recent starburst. Dolphin et al. (2001) and Izotov \& Thuan (2002) resolved UGC 4483 into single stars and showed that the young stellar populations are mostly concentrated to the North. The mass in young stars, however, constitutes only $\sim 10 \%$ of the total stellar mass: the SFH derived by McQuinn et al. (2010a) (modeling the color-magnitude diagram) implies that the stellar mass formed in the last $500 \mathrm{Myr}$ is $\sim 0.15 \times 10^{7} \mathrm{M}_{\odot}$, whereas the stellar mass formed more than $500 \mathrm{Myr}$ ago is $\sim 1.31 \times 10^{7} M_{\odot}$. Thus, to calculate the gravitational contribution of the stars, we used the southern surface brightness profile, as it provides a better approximation of the stellar mass surface density. Assuming that $30 \% \pm 10 \%$ of the gas is returned to the inter stellar medium by supernovae and stellar winds, the total stellar mass is $(1.0 \pm 0.3) \times 10^{7} M_{\odot}($ cf. McQuinn et al. 2010b), giving a stellar mass-to-light ratio $M_{*} / L_{R}=1.1 \pm 0.3$. The southern surface brightness profile can be fitted by an exponential-law with $\mu_{0}=22.0 \pm 0.1 \mathrm{mag} \operatorname{arcsec}^{-2}\left(\sim 40 L_{\odot} \mathrm{pc}^{-2}\right)$ and $R_{0}=13.1^{\prime \prime} \pm$ $0.1^{\prime \prime}(\sim 203 \mathrm{pc})$. We assumed that the stars are located in a disk with vertical density distribution given by $\rho(z)=\operatorname{sech}^{2}\left(z / z_{0}\right)$ (van der Kruit $\&$ Searle 1981) with $z_{0} \simeq 0.5 R_{0} \simeq 100 \mathrm{pc}$.

The gravitational contribution of the gaseous disk was calculated using the azimuthally-averaged H I surface density profile, which was derived from the total H I map. Figure 6 shows the $\mathrm{HI}$ density profiles derived by azimuthally-averaging over the entire galaxy and over the southern (approaching) and northern (receding) sides separately. There is a difference on the order of $\sim 2-3 M_{\odot} \mathrm{pc}^{-2}$ between the two halves, that has a small effect on the resulting gravitational contribution. In agreement with the models in Sect. 3.2, we assumed an exponential vertical distribution with $z_{0}=100 \mathrm{pc}$. The mass of the H I disk was calculated from the total H I flux (see Table 1) and multiplied by a factor of 1.32 to take into account the presence of helium. Molecular gas was not explicitly considered in the mass model because its amount is unknown.

For the dark matter distribution, we assumed a pseudoisothermal halo described by the equation

$\rho_{\mathrm{ISO}}(r)=\frac{\rho_{0}}{1+\left(r / r_{\mathrm{c}}\right)^{2}}$,

where the central density $\rho_{0}$ and the core radius $r_{\mathrm{c}}$ are both free parameters of the mass model.

Figure 8 (left) shows the rotation curve decomposition assuming that $M_{*} / L_{R}=1.1$, as is found by integrating the galaxy SFH (McQuinn et al. 2010a,b). The gravitational contribution of the stars $V_{*}$ is $\sim 50 \%$ of the circular velocity $V_{\text {circ }}$ at $R_{\text {peak }}=2.2 R_{0} \simeq 450 \mathrm{pc}$. The parameters of the halo are: $\rho_{0}=(101 \pm 20) \times 10^{-3} M_{\odot} \mathrm{pc}^{-3}$ and $r_{\mathrm{c}}=0.34 \pm 0.05 \mathrm{kpc}$. These values are comparable with those found by Swaters et al. (2011) for a sample of $18 \mathrm{dIrrs}$ (assuming that $M_{*} / L_{R}=1$ ); the halo of UGC 4483, however, is one with the smallest core radius and highest central density. As discussed at the end of Sect. 3.1, we applied the asymmetric-drift correction assuming a constant velocity dispersion $\sigma_{\mathrm{HI}}=8 \mathrm{~km} \mathrm{~s}^{-1}$. Different values of $\sigma_{\mathrm{HI}}$ would change the circular velocity at large radii and thus give slightly 
different values for the dark matter halo. In particular, considering that $\sigma_{\mathrm{H} \mathrm{I}}$ may be between 6 and $8 \mathrm{~km} \mathrm{~s}^{-1}$, the dynamical mass within the last measured point of the rotation curve may be between 1 and $2 \times 10^{8} M_{\odot}$ and the baryon fraction between $\sim 43 \%$ and $\sim 22 \%$, respectively. In any case, baryons (gas and stars) constitute a relevant fraction of the total mass.

A stellar disk is defined to be maximum if $F_{*}=V_{*} / V_{\text {circ }}=$ $0.85 \pm 0.10$ at $R_{\text {peak }}$ (Sackett 1997; Bershady et al. 2011). In our case $F_{*}=0.5 \pm 0.1$; the error takes into account the uncertainties on the stellar mass, the gas-recycling efficiency and the rotation velocity. This is in line with the results of the DiskMass survey (Bershady et al. 2011), that have measured the stellar velocity dispersion in a sample of spiral galaxies and found that stellar disks typically have $F_{*} \sim 0.5$. We point out that the color-magnitude diagrams of the resolved stellar populations provide the most direct method to quantify stellar masses, as the results depend only slightly on the assumed evolutionary tracks, metallicity, and sampling of the SFH (e.g. Annibali et al. 2003). The initial mass function (IMF), instead, may have a stronger effect: McQuinn et al. (2010a) assumed a single-slope Salpeter from 0.1 to $100 M_{\odot}$ and a binary fraction of $35 \%$. If the Chabrier and/or Kroupa IMFs are assumed, the stellar mass would systematically decrease by, respectively, $\sim 25 \%$ and $\sim 30 \%$ and the gas-recycling efficiency may go up to $\sim 50 \%$, further decreasing the stellar mass.

We note that the molecular gas component has been neglected so far. UGC 4483 is undetected in the CO line with an upper limit of $0.195 \mathrm{~K} \mathrm{~km} \mathrm{~s}^{-1}$ within the inner $55^{\prime \prime}(\sim 800 \mathrm{pc})$ (Taylor et al. 1998). We extrapolated the relation between the CO-to- $\mathrm{H}_{2}$ conversion factor and metallicity from Boselli et al. (2002) down to the metallicity of UGC 4483 and obtained a corresponding upper limit on the $\mathrm{H}_{2}$ mass of $M_{\mathrm{H}_{2}} \lesssim 10^{7} M_{\odot}$. Alternatively, we estimated the $\mathrm{H}_{2}$ mass from the empirical relation between SFR and molecular mass, as determined by Leroy et al. (2008), although this relation may not apply to starburst galaxies. Assuming that $S F R=0.01 M_{\odot} \mathrm{yr}^{-1}$ (McQuinn et al. 2010a), we derived $M_{\mathrm{H}_{2}} \simeq 1.9 \times 10^{7} M_{\odot}$. Both estimates indicate that the molecular gas may be dynamically important and comparable in mass to the stellar component, implying a full maximum-disk situation, as we now discuss.

Figure 8 (right) shows the maximum-disk solution (Sancisi \& van Albada 1987). The stellar contribution can explain the inner parts of the rotation curve if $M_{*} / L_{R} \simeq 3.3$, i.e. if the stellar mass is $\sim 3$ times higher than obtained from the study of the resolved stellar populations. This mass may be provided by molecules, if they are distributed in a way similar to the stars. We also find a good fit by fixing $M_{*} / L_{R}=1.1$ and scaling the H I contribution by a factor of $\sim 5$, similarly to the results of Hoekstra et al. (2001) for other gas-rich galaxies. These results suggest that the distribution of the dynamical mass is closely coupled to that of the baryonic mass (see e.g. Sancisi 2004; Swaters et al. 2011). We also checked the position of UGC 4483 on the baryonic Tully-Fisher relation and found that it follows the correlation within the observed scatter (cf. McGaugh 2011).

Finally, we considered the predictions of the MOdified Newtonian Dynamics (MOND; Milgrom 1983; see Famaey \& McGaugh 2011, for a review). Using UGC 4483, we can test MOND without any free parameter, as the value of $M_{*} / L$ is provided by the color-magnitude diagram of the resolved stellar populations and the distance is well determined from the tip of the red giant branch (Dolphin et al. 2001; Izotov \& Thuan 2002). We found that MOND systematically over-predicts the observed rotation curve by $\sim 5-6 \mathrm{~km} \mathrm{~s}^{-1}$. The discrepancy does not strongly depend on the assumed $M_{*} / L$, as it is mainly driven

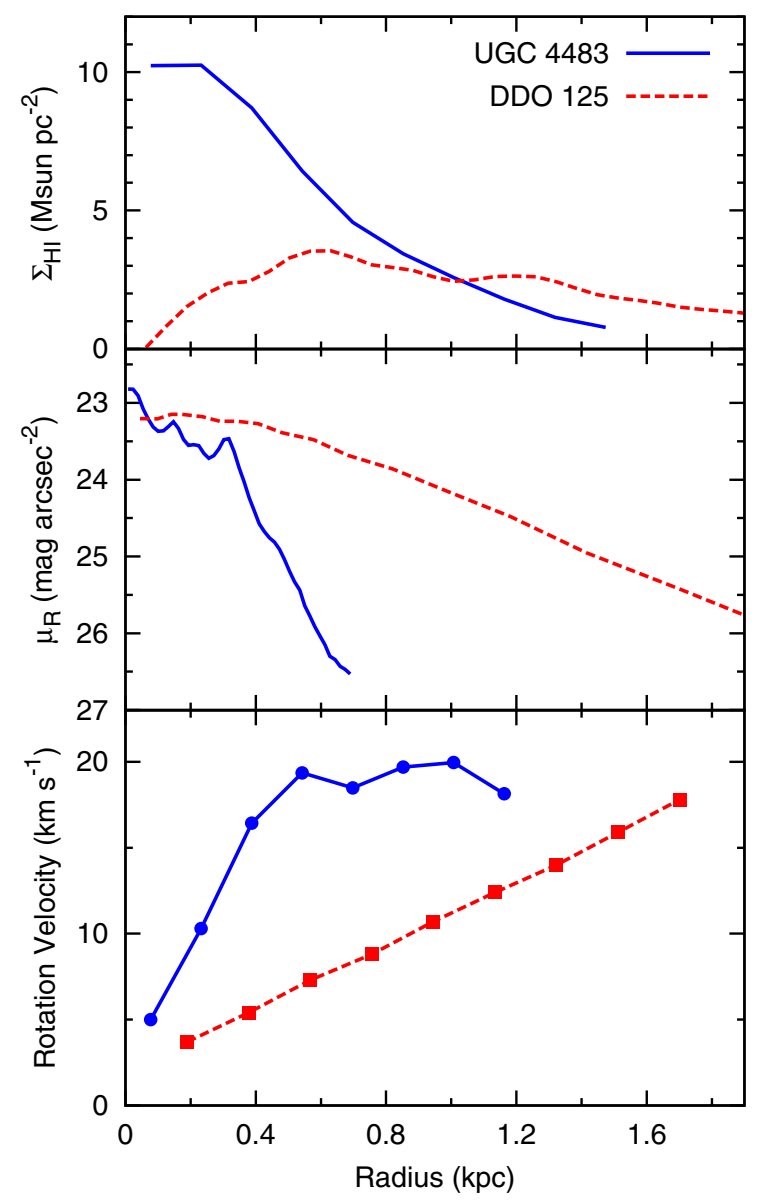

Fig. 9. Comparison between UGC 4483 (blue line) and the dwarf irregular galaxy DDO 125 (UGC 7577) (red line). Top: H I surface density profiles. Middle: $R$-band surface brightness profiles, corrected for the inclination assuming that the disk is transparent and using the ellipticity from Swaters \& Balcells (2002). Bottom: H I rotation curves (not corrected for asymmetric drift).

by the atomic gas content, and it would further increase if molecules are also considered in the mass model. However, the discrepancy disappears if the inclination of the H I disk is assumed to be $\sim 43^{\circ}$ instead of $58^{\circ}$ (as is derived from the optical image). This possibility cannot be ruled out by the present observations, because it is not possible to determine $i$ neither from the total H I map (as the H I distribution is strongly lopsided) nor from the velocity field.

\section{Discussion}

In Sect. 3.2, we showed that UGC 4483 has a steeply-rising rotation curve that flattens in the outer parts at a velocity of $\sim 18-20 \mathrm{~km} \mathrm{~s}^{-1}$. As far as we know, this is the lowest-mass galaxy with a differentially rotating $\mathrm{H}$ I disk. The steep rise of the rotation curve points to a strong central concentration of mass, which seems to be a characterizing property of BCDs. Indeed, similar results have been found also for other BCDs, e.g. I Zw 18 (Lelli et al. 2012), NGC 2537 (Matthews \& Uson 2008), and NGC 1705 (Meurer et al. 1998). The steeply-rising and flat rotation curves of these BCDs are remarkable, as dIrrs typically have slowly-rising rotation curves (e.g. Swaters et al. 2009). The terms "steeply-rising" and "slowly-rising" are referred to rotation-velocity gradients measured in physical units $\left(\mathrm{km} \mathrm{s}^{-1} \mathrm{kpc}^{-1}\right)$ that can be directly related to the dynamical mass 
F. Lelli et al.: Dynamics of starbursting dwarf galaxies. II.
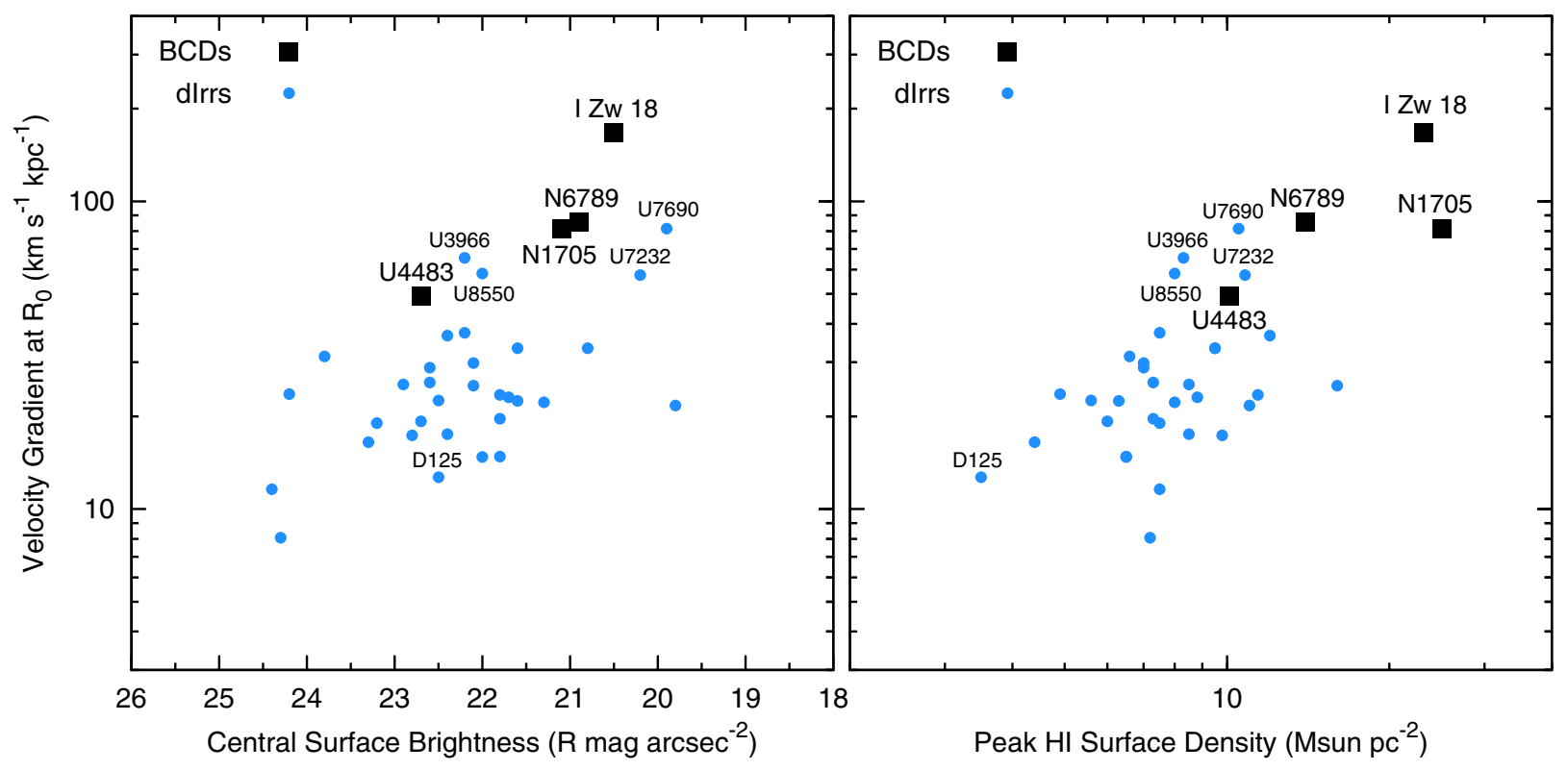

Fig. 10. Comparison between the properties of BCDs (squares) and of dIrrs (dots). The dIrrs are taken from Swaters et al. (2009), selecting the objects with high-quality rotation curves and $V_{\text {rot }}<70 \mathrm{~km} \mathrm{~s}^{-1}$ at the last measured point. The BCDs are UGC 4483 (this work), I Zw 18 (Lelli et al. 2012), NGC 6789 (Lelli et al., in prep.), and NGC 1705 (Meurer et al. 1998). Some galaxies are labelled according to their UGC number. Left: $R$-band central surface brightness (inclination corrected) versus the inner rotation-velocity gradient $V_{\text {rot }}\left(R_{0}\right) / R_{0}$, where $R_{0}$ is the optical scalelength. Right: peak H I surface density $\Sigma_{\mathrm{HI} \text {, peak }}$ versus $V_{\mathrm{rot}}\left(R_{0}\right) / R_{0} . \Sigma_{\mathrm{HI} \text {, peak }}$ is derived from the azimuthally-averaged H I surface density profiles. See Sect. 4 for details.

surface densities in $M_{\odot} \mathrm{pc}^{-2}$. If these gradients are expressed in terms of disk scale-lengths, rotation curves of dIrrs rise as steep as those of spiral galaxies (e.g. Swaters et al. 2009).

In Lelli et al. (2012, Fig. 10), we have illustrated the dynamical difference between the BCD-prototype I Zw 18 and a typical dIrr of the same mass. Similarly, Fig. 9 compares UGC 4483 with the dwarf irregular DDO 125 (UGC 7577, from Swaters et al. 2009). Assuming that DDO 125 is at a distance of $2.6 \mathrm{Mpc}$ (Jacobs et al. 2009; Dalcanton et al. 2009), the H I observations of Swaters et al. (2009) have a linear resolution of $\sim 180 \mathrm{pc}$, similar to our linear resolution for UGC $4483(\sim 155 \mathrm{pc})$, making it possible to compare H I surface densities and velocity gradients. The two galaxies have approximately the same rotation velocity at the last measured point $\left(\sim 20 \mathrm{~km} \mathrm{~s}^{-1}\right)$ and dynamical mass $\left(\sim 1-2 \times 10^{8} M_{\odot}\right)$, but their structural properties are very different: i) the HI surface densities of UGC 4483 are $\sim 3-4$ times higher than those of DDO 125 in the inner regions (top); ii) the stellar component of UGC 4483 is much more compact than that of DDO 125 (middle); iii) the rotation curve of UGC 4483 has a steeper rise than the one of DDO 125 and flattens in the outer parts. These structural and dynamical differences between BCDs and typical dIrrs must be the key to understand the starburst phenomenon in BCDs. They also shed new light on the question of the progenitors and descendants of BCDs, as we now discuss.

In Sect. 3.3, we showed that the central mass concentration of UGC 4483 cannot be explained by the newly formed stars or by the concentration of HI. Old stars, although not sufficient to account for the inner rise of the rotation curve, constitute an important fraction of the mass in the inner parts. Similarly, the central mass concentration of I Zw 18 may be due to old stars and/or dark matter (unfortunately, the HST observations of I Zw 18 do not provide a direct estimate of the mass in old stars, see Contreras Ramos et al. 2011). For both galaxies, the gravitational contribution of molecules is very uncertain and it is unclear whether they are dynamically important or not. These results imply that either the progenitors of these BCDs are unusually compact, gas-rich dwarfs, or there must be a mechanism that leads to a concentration of gas, old stars, and/or dark matter, eventually causing the starburst. This mechanism may be external (interactions/mergers) and/or internal (torques from massive star-forming "clumps", see Elmegreen et al. 2012). It is also clear that, unless a redistribution of mass takes place, the descendants of BCDs must be compact dwarfs. Compact dIrrs do exist and have the following properties: i) high-surface-brightness exponential profiles (see e.g. UGC 7690 and UGC 8550 in Swaters \& Balcells 2002) or low-surfacebrightness exponential profiles with an inner light concentration (see e.g. UGC 6628 and UGC 12632 in Swaters \& Balcells 2002); and ii) steeply-rising and flat rotation curves (see the same galaxies in Swaters et al. 2009). The evolutionary links outlined here are in line with the results of e.g. Papaderos et al. (1996) and Gil de Paz \& Madore (2005), which are based on surface photometry of large galaxy samples and indicate that the old stellar component of BCDs generally has higher central surface brightness and smaller scale-length than typical dIrrs and dSphs/dEs. We present here two plots that compare the structural and dynamical properties of BCDs and dIrrs.

To compare the dynamics of BCDs and dIrrs, we use the inner rotation-velocity gradient $V_{\text {rot }}(R) / R$ as an estimate of central mass concentration. This is calculated at $R=R_{0}$, where $R_{0}$ is the galaxy scale-length in the $R$-band; thus we take into account the different sizes of the systems. In Fig. 10, $V_{\text {rot }}\left(R_{0}\right) / R_{0}$ is plotted versus the central disk surface brightness $\mu_{0}$ (left) and the peak H I column density $\Sigma_{\mathrm{HI}}$, peak (right) for a sample of both BCDs and dIrrs. The BCDs are UGC 4483, I Zw 18, NGC 1705, and NGC 6789. For I Zw 18 we used the $R$-band structural parameters from Papaderos et al. (2002), the rotation curve from Lelli et al. (2012), and a distance of $18.2 \mathrm{Mpc}$ (Aloisi et al. 2007). For NGC 1705 we used the $R$-band structural parameters from Gil de Paz \& Madore (2005), the rotation curve from Meurer et al. (1998) named "model DD" (see 
their Fig. 10), and a distance of 5.1 Mpc (Tosi et al. 2001). For NGC 6789 we used the $R$-band structural parameters and the rotation curve from Lelli et al. (in prep.), and a distance of 3.6 Mpc (Drozdovsky et al. 2001). The dIrrs were taken from the sample of Swaters et al. (2009), by selecting the objects that have high-quality rotation curves $(q \leq 2$, see Swaters et al. 2009) and $V_{\text {rot }}<70 \mathrm{~km} \mathrm{~s}^{-1}$ at the last measured point. For $\Sigma_{\mathrm{HI}}$, peak, we used the peak value of the azimuthally-averaged HI density profile. The value of $\Sigma_{\mathrm{HI}}$, peak depends on the linear resolution of the H I observations, but we checked that this does not strongly bias our analysis as the trend in Fig. 10 does not significantly change if one considers only galaxies with linear resolution $\lesssim 500 \mathrm{pc}$ (corresponding to distances $\lesssim 7 \mathrm{Mpc}$ ). We also point out that BCDs usually have clumpy H I distributions and locally the H I column densities can reach even higher values, up to $40-50 M_{\odot} \mathrm{pc}^{-2}$, much higher than in dIrrs.

The two diagrams in Fig. 10 show a clear trend: galaxies with a high rotation-velocity gradient have also high central surface brightness (left) and high peak H I surface density (right). The BCDs are in the upper parts of these distributions: they have $V_{\text {rot }}\left(R_{0}\right) / R_{0}>40 \mathrm{~km} \mathrm{~s}^{-1} \mathrm{kpc}^{-1}$ whereas dIrrs typically have $V_{\text {rot }}\left(R_{0}\right) / R_{0}<40 \mathrm{~km} \mathrm{~s}^{-1} \mathrm{kpc}^{-1}$. However, some dIrrs (UGC 3966, UGC 8550, UGC 7232, and UGC 7690) have inner rotation-velocity gradients comparable to those of BCDs and thus, in this respect, are dynamically similar to BCDs. These objects have peak HI column densities much smaller than those found in I Zw 18 and NGC 1705 (Fig. 10, right) and are candidate progenitors/descendants of BCDs. It would be interesting to study the SFHs of these compact dIrrs and investigate if they experienced a starburst in the recent past.

Finally, we have seen that the asymmetries in the velocity field of UGC 4483 can be described as a global radial motion of $5 \mathrm{~km} \mathrm{~s}^{-1}$. Radial motions have been found in two other BCDs: NGC 2915 (Elson et al. 2011a,b) and I Zw 18 (Lelli et al. 2012). Elson et al. (2011a) assumed that the spiral arms in the H I disk of NGC 2915 are trailing and concluded that the radial motions are an outflow. For I Zw 18 and UGC 4483, it is not possible to discriminate between inflow and outflow, as it is not known which side of the disk is the near one. For both galaxies, we calculated the timescales associated with the radial motions $\left(\simeq R_{\mathrm{HI}} / V_{\mathrm{rad}}\right)$ and found that they are on the same order of magnitude of the orbital times $\left(\simeq 2 \pi R_{\mathrm{HI}} / V_{\text {rot }}\right)$. This suggests that any outflow or inflow must be very recent and possibly associated with the most recent burst of star-formation ( 10-20 Myr, see McQuinn et al. 2010a, for UGC 4483; and Aloisi et al. 1999, for I $\mathrm{Zw} \mathrm{18).} \mathrm{If} \mathrm{the} \mathrm{radial} \mathrm{motions} \mathrm{were} \mathrm{an} \mathrm{outflow,} \mathrm{its} \mathrm{kinetic}$ energy would correspond to only $\sim 1 \%$ of the energy released by supernovae.

\section{Conclusions}

We analysed archival H I observations of the blue compact dwarf galaxy UGC 4483 and built model datacubes to investigate its gas kinematics. Our main results can be summarized as follows:

- UGC 4483 has a steeply-rising rotation curve that flattens in the outer parts at a velocity of $\sim 20 \mathrm{~km} \mathrm{~s}^{-1}$. This is, to our knowledge, the lowest-mass galaxy with a differentially rotating H I disk. Radial motions of $\sim 5 \mathrm{~km} \mathrm{~s}^{-1}$ may also be present.

- The steep rise of the rotation curve indicates that there is a strong central concentration of mass. Mass models with a dark matter halo show that old stars contribute $\sim 50 \%$ of the observed rotation speed at 2.2 disk scale-lengths. Baryons (gas and stars) constitute an important fraction of the total dynamical mass. These conclusions are based on the stellar mass obtained from the color-magnitude diagram of the resolved stellar populations.

- The maximum-disk solution requires a stellar mass 3 times higher than observed, that could be provided by molecules. A good solution is also found by scaling the H I contribution by a factor of $\sim 5$. These results suggest that the distribution of the dynamical mass is closely coupled to that of the baryons.

UGC 4483, together with other BCDs like I Zw 18 and NGC 1705, appears structurally different from typical dIrrs in terms of H I distribution, stellar distribution, and dynamics. In particular, a central concentration of mass (gas, stars, and dark matter) seems to be a characterizing property of BCDs. This implies that the starburst is closely related with the gravitational potential and the $\mathrm{HI}$ concentration. Our results also suggest that the progenitors/descendants of BCDs must be compact dwarf galaxies, unless a redistribution of mass (both luminous and dark) takes place before/after the starbursting phase.

Acknowledgements. We thank Rob Swaters for stimulating comments. We are grateful to F. Annibali and M. Tosi for helpful discussions about the stellar populations of BCDs. We thank the ISSI (Bern) for support of the team "Defining the full life-cycle of dwarf galaxy evolution: the Local Universe as a template".

\section{References}

Aloisi, A., Tosi, M., \& Greggio, L. 1999, AJ, 118, 302

Aloisi, A., Clementini, G., Tosi, M., et al. 2007, ApJ, 667, L151

Annibali, F., Greggio, L., Tosi, M., Aloisi, A., \& Leitherer, C. 2003, AJ, 126, 2752

Begeman, K. G. 1987, Ph.D. Thesis, Kapteyn Institute

Bershady, M. A., Martinsson, T. P. K., Verheijen, M. A. W., et al. 2011, ApJ, 739, L47

Binney, J., \& Merrifield, M. 1998, Galactic astronomy, eds. J. Binney, \& M. Merrifield

Boselli, A., Lequeux, J., \& Gavazzi, G. 2002, A\&A, 384, 33

Bosma, A. 1978, Ph.D. Thesis, Groningen Univ.

Briggs, D. S. 1995, in BAAS, 27, 1444

Contreras Ramos, R., Annibali, F., Fiorentino, G., et al. 2011, ApJ, 739, 74

Dalcanton, J. J., Williams, B. F., Seth, A. C., et al. 2009, ApJS, 183, 67

Dolphin, A. E., Makarova, L., Karachentsev, I. D., et al. 2001, MNRAS, 324, 249

Drozdovsky, I. O., Schulte-Ladbeck, R. E., Hopp, U., Crone, M. M., \& Greggio, L. 2001, ApJ, 551, L135

Elmegreen, B. G., Zhang, H.-X., \& Hunter, D. A. 2012, ApJ, 747, 105

Elson, E. C., de Blok, W. J. G., \& Kraan-Korteweg, R. C. 2011a, MNRAS, 411, 200

Elson, E. C., de Blok, W. J. G., \& Kraan-Korteweg, R. C. 2011 b, MNRAS, 415, 323

Famaey, B., \& McGaugh, S. 2011 [arXiv: 1112.3960]

Fraternali, F., van Moorsel, G., Sancisi, R., \& Oosterloo, T. 2002, AJ, 123, 3124

Gallagher, III, J. S., \& Hunter, D. A. 1987, AJ, 94, 43

Gil de Paz, A., \& Madore, B. F. 2005, ApJS, 156, 345

Gil de Paz, A., Madore, B. F., \& Pevunova, O. 2003, ApJS, 147, 29

Hoekstra, H., van Albada, T. S., \& Sancisi, R. 2001, MNRAS, 323, 453

Högbom, J. A. 1974, A\&AS, 15, 417

Izotov, Y. I., \& Thuan, T. X. 2002, ApJ, 567, 875

Jacobs, B. A., Rizzi, L., Tully, R. B., et al. 2009, AJ, 138, 332

Lelli, F., Fraternali, F., \& Sancisi, R. 2010, A\&A, 516, A11

Lelli, F., Verheijen, M., Fraternali, F., \& Sancisi, R. 2012, A\&A, 537, A72

Leroy, A. K., Walter, F., Brinks, E., et al. 2008, AJ, 136, 2782

Lo, K. Y., Sargent, W. L. W., \& Young, K. 1993, AJ, 106, 507

Matthews, L. D., \& Uson, J. M. 2008, AJ, 135, 291

McGaugh, S. S. 2011, Phys. Rev. Lett., 106, 121303

McQuinn, K. B. W., Skillman, E. D., Cannon, J. M., et al. 2010a, ApJ, 721, 297 McQuinn, K. B. W., Skillman, E. D., Cannon, J. M., et al. 2010b, ApJ, 724, 49

Meurer, G. R., Carignan, C., Beaulieu, S. F., \& Freeman, K. C. 1996, AJ, 111, 1551

Meurer, G. R., Staveley-Smith, L., \& Killeen, N. E. B. 1998, MNRAS, 300, 705 Milgrom, M. 1983, MNRAS, 270, 365

Papaderos, P., Loose, H., Fricke, K. J., \& Thuan, T. X. 1996, A\&A, 314, 59 
F. Lelli et al.: Dynamics of starbursting dwarf galaxies. II.

Papaderos, P., Izotov, Y. I., Thuan, T. X., et al. 2002, A\&A, 393, 461

Sackett, P. D. 1997, ApJ, 483, 103

Sancisi, R. 2004, in Dark Matter in Galaxies, eds. S. Ryder, D. Pisano, M. Walker, \& K. Freeman, IAU Symp., 220, 233

Sancisi, R., \& van Albada, T. S. 1987, in Dark matter in the universe, eds. J. Kormendy, \& G. R. Knapp, IAU Symp., 117, 67

Schlegel, D. J., Finkbeiner, D. P., \& Davis, M. 1998, ApJ, 500, 525

Searle, L., \& Sargent, W. L. W. 1972, ApJ, 173, 25

Skillman, E. D., Televich, R. J., Kennicutt, Jr., R. C., Garnett, D. R., \& Terlevich, E. 1994, ApJ, 431, 172

Swaters, R. A., \& Balcells, M. 2002, A\&A, 390, 863

Swaters, R. A., Sancisi, R., van Albada, T. S., \& van der Hulst, J. M. 2009, A\&A, 493, 871

Swaters, R. A., Sancisi, R., van Albada, T. S., \& van der Hulst, J. M. 2011, ApJ, 729,118
Taylor, C. L., Brinks, E., Grashuis, R. M., \& Skillman, E. D. 1995, ApJS, 99, 427

Taylor, C. L., Kobulnicky, H. A., \& Skillman, E. D. 1998, AJ, 116, 2746

Tosi, M. 2009, in IAU Symp. 258, eds. E. E. Mamajek, D. R. Soderblom, \& R. F. G. Wyse, 61

Tosi, M., Sabbi, E., Bellazzini, M., et al. 2001, AJ, 122, 1271

van der Hulst, J., Terlouw, J., Begeman, K., Zwitser, W., \& Roelfsema, P. 1992, in ASP Conf. Ser. 25, eds. D. M. Worall, C. Biemesderfer, \& J. Barnes (San Francisco: ASP), 131

van der Kruit, P. C., \& Searle, L. 1981, A\&A, 95, 105

van Zee, L., \& Haynes, M. P. 2006, ApJ, 636, 214

van Zee, L., Skillman, E. D., \& Salzer, J. J. 1998, AJ, 116, 1186

van Zee, L., Salzer, J. J., \& Skillman, E. D. 2001, AJ, 122, 121

Verheijen, M., \& Sancisi, R. 2001, A\&A, 370, 765

Warner, P. J., Wright, M. C. H., \& Baldwin, J. E. 1973, MNRAS, 163, 163 